\title{
Changes of soluble CD40 ligand in the progression of acute myocardial infarction associate to endothelial nitric oxide synthase polymorphisms and vascular endothelial growth factor but not to platelet CD62P expression
}

\author{
PATRÍCIA NAPOLEÃO, MARIA DO CÉU MONTEIRO, LUÍS B. P. CABRAL, \\ MARIA BEGOÑA CRIADO, CATARINA RAMOS, MAFALDA SELAS, \\ ANA MARIA VIEGAS-CRESPO, CARLOTA SALDANHA, MIGUEL MOTA CARMO, \\ RUI CRUZ FERREIRA, and TERESA PINHEIRO
}

LISBOA AND PENAFIEL, PORTUGAL

\begin{abstract}
Reported in vitro data implicated soluble CD40 ligand (SCD40L) in endothelial dysfunction and angiogenesis. However, whether SCD4OL could exert that influence in endothelial dysfunction and angiogenesis after injury in acute myocardial infarction (AMI) patients remains unclear. In the present study, we evaluated the association of SCD40L with markers of platelet activation, endothelial, and vascular function during a recovery period early after AMI. To achieve this goal, the time changes of soluble, platelet-bound, and microparticle-bound CD4OL levels over 1 month were assessed in AMI patients and correlated with endothelial nitric oxide synthase (eNOS) polymorphisms, vascular endothelial growth factor (VEGF) concentrations, and platelet expression of P-selectin (CD62P). The association of soluble form, platelet-bound, and microparticle-bound CD40L with CD62P expression on platelets, a marker of platelet activation, was also assessed to evaluate the role of CD40L in the thrombosis, whereas the association with eNOS and VEGF was to evaluate the role of $\mathrm{CD} 40 \mathrm{~L}$ in vascular dysfunction. This work shows for the first time that time changes of SCD40L over 1 month after myocardial infarct onset were associated with G894T eNOS polymorphism and with the VEGF concentrations, but not to the platelet CD62P expression. These results indicate that, in terms of AMI pathophysiology, the SCD40L cannot be consider just as being involved in thrombosis and inflammation but also as having a relevant role in vascular and endothelial dysfunction. (Translational Research 2015;166:650-659)
\end{abstract}

From the Carlota Saldanha Lab, Instituto Medicina Molecular (iMM), Faculdade de Medicina da Universidade de Lisboa, Lisboa, Portugal; IINFACTS-CESPU, Instituto de Investigação e Formação Avançada em Ciências e Tecnologias da Saúde, Penafiel, Portugal; Departamento de Engenharia e Ciências Nucleares, Instituto de Bioengenharia e Biociências (IBB), Instituto Superior Técnico, Universidade de Lisboa, Portugal; Serviço Cardiologia, Hospital Santa Marta, Centro Hospitalar Lisboa Central (CHLC), Lisboa, Portugal; Centro de Estudos do Ambiente e do Mar (CESAM) \& Departamento de Biologia Animal (DBA), Faculdade de Ciências da Universidade de Lisboa, Lisboa, Portugal; Centro de Estudos de
Doenças Crónicas (CEDOC), Faculdade Ciências Médias, Universidade Nova de Lisboa, Lisboa, Portugal.

Submitted for publication March 31, 2015; revision submitted June 19, 2015; accepted for publication July 24, 2015

Reprint requests: Patrícia Napoleão, Carlota Saldanha Lab, Instituto de Medicina Molecular, Av. Professor Egas Moniz, 1649-028 Lisboa, Portugal; e-mail: pnapoleao@medicina.ulisboa.pt.

1931-5244/\$ - see front matter

(C) 2015 Elsevier Inc. All rights reserved.

http://dx.doi.org/10.1016/j.trsl.2015.07.006 


\begin{abstract}
Abbreviations: $\mathrm{AMI}=$ acute myocardial infarction; $\mathrm{APC}=$ allophycocyanin; $\mathrm{Asp}=$ aspartate $\mathrm{CTNT}=$ cardiac troponin; $\mathrm{CAD}=$ coronary artery disease; $\mathrm{CRP}=\mathrm{C}$-reactive protein; $\mathrm{CK}=\mathrm{cre}$ atine kinase; eNOS = endothelial nitric oxide synthase; ELISA = enzyme-linked immunosorbent assay; FITC = fluorescein isothiocyanate; FAU = fluorescence arbitrary units; Glu = glutamic acid; $L M E$ = linear mixed effects model; MPs = microparticles; NO = nitric oxide; NT-proBNP = $\mathrm{N}$-terminal pro-brain natriuretic peptide; $\mathrm{PCl}=$ percutaneous coronary intervention; $\mathrm{PBS}=$ phosphate-buffered saline; $\mathrm{PE}=$ Phycoerythrin; $\mathrm{CD62P}=$ P-selectin; $\mathrm{sCD} 40 \mathrm{~L}=$ soluble CD40 ligand; $S A=$ stable angina pectoris; VEGF = vascular endothelial growth factor
\end{abstract}

\section{AT A GLANCE COMMENTARY}

\section{Napoleão $P$, et al.}

\section{Background}

The influence of sCD40L in endothelial dysfunction and angiogenesis markers in AMI patients remains unclear. The influence of sCD40L in endothelial and vascular function in early stages after AMI was studied having platelet activation into account.

\section{Translational Significance}

It was found for the first time that time changes of sCD40L in AMI patients were associated with G894T eNOS polymorphism and VEGF concentrations, but not to the platelet P-selectin expression. In terms of AMI pathophysiology, sCD40L cannot be considered just as a marker of thrombosis and inflammation but has also a relevant role in vascular and endothelial dysfunction. A prognostic value for the sCD40L could be hypothesized along the progression of the disease in AMI patients. This may be of the utmost importance in clinical diagnostic of AMI.

\section{INTRODUCTION}

$\mathrm{CD} 40 \mathrm{~L}$ is a signaling molecule, ${ }^{1-3}$ implicated in thrombosis and inflammatory response to vascular injury. ${ }^{4-6}$ The relationship of CD40L with coronary artery disease (CAD) has been established,,$^{2,7-9}$ as also its implication in endothelial dysfunction. ${ }^{10-14}$ However, whether the soluble CD40 ligand (sCD40L) could also influence endothelial dysfunction after acute myocardial infarction (AMI) injury remains unclear.

In vitro studies have shown that sCD40L inhibits angiogenesis and also growth factor-induced human umbilical vein endothelial cell migration, which is achieved by generation of free radicals and inhibition of nitric oxide (NO) production. ${ }^{10}$ The authors hypothe- sized that the $\mathrm{SCD} 40 \mathrm{~L}$ could inhibit reendothelialization of an injured vessel, thereby affecting the restenosis. ${ }^{10}$

Research efforts have been directed toward the finding of biomarkers to assess endothelial function and its correlation with AMI. Genetic indicators, such as the polymorphisms of endothelial NO synthase (eNOS) gene, ${ }^{15,16}$ may provide insight into endothelial cells function.

Vascular endothelial growth factor (VEGF) is a wellknown promoter of angiogenesis and an endogenous regulator of endothelial integrity. ${ }^{17-19}$ The prognostic information provided by VEGF independently of other markers likely points toward an important role for angiogenesis in regulating myocardial repair and reperfusion after AMI. ${ }^{17,20}$

Current opinion suggests a differential role of CD40L (both soluble and membrane-bound forms, which includes microparticles in circulation) ${ }^{21}$ at different stages of $\mathrm{CAD}$, contrasting with the traditional view of an unvarying function of the CD40L-CD40-sCD40L system interactions in the disease. ${ }^{6}$ In that perspective, no clear indication of the interplay of CD40L with endothelial and vascular function markers and their importance in the pathophysiology of the AMI has been obtained so far in human clinical studies. Therefore, the aim of this study was to evaluate the relationship of SCD40L with markers of platelet activation, endothelial and vascular function during an early recovery period after AMI. To achieve this goal, the time changes over 1 month of sCD40L levels were assessed in AMI patients and correlated with the CD40L expressed on platelets and microparticles, CD62P expression on platelets, and eNOS polymorphisms VEGF concentrations. The association of soluble form, platelet-bound, and microparticle-bound CD40L with CD62P expression on platelets was assessed to evaluate the role of CD40L in thrombosis, whereas the association with eNOS and VEGF was to evaluate the role of CD40L in vascular dysfunction. The sCD40L serum concentrations were measured and compared with the expression of CD40L on platelets and microparticles. Healthy volunteers (CTR) and longitudinally assessed stable angina (SA) patients were used as predictors of altered endothelial regulation in AMI. 
Table I. Baseline demographic and clinical characteristics of the study population

\begin{tabular}{|c|c|c|c|}
\hline & CTR $(n=63)$ & $\mathrm{SA}(n=48)$ & AMI $(n=89)$ \\
\hline $\operatorname{Sex}(f / m)$ & $23 / 40$ & $13 / 35$ & $21 / 67$ \\
\hline Age $(y)$ & $55(47-65)$ & $63(57-73)$ & $63(54-72)$ \\
\hline $\mathrm{BMI}\left(\mathrm{kg} / \mathrm{m}^{2}\right)$ & $25(24-28)$ & $28(25-29)$ & $27(24-30)$ \\
\hline Waist perimeter $(\mathrm{cm})$ & $86(82-94)$ & $96(91-102)$ & $99(89-106)$ \\
\hline \multicolumn{4}{|l|}{ Genotyping polymorphisms } \\
\hline eNOS G894T (GG/T), \% & $38 / 62$ & $37 / 63$ & $38 / 63$ \\
\hline eNOS T786C (TT/C), \% & $43 / 57$ & $50 / 50$ & $28 / 72^{*}$ \\
\hline \multicolumn{4}{|l|}{ Risk factors and comorbidity } \\
\hline Hypertension, n (\%) & $18(29)$ & $37(77)^{\dagger}$ & $58(65)^{\dagger}$ \\
\hline Hyperlipidemia, n (\%) & $29(46)$ & $35(73)^{\dagger}$ & $47(53)^{\star}$ \\
\hline Diabetes, n (\%) & $2(3)$ & $16(33)^{\dagger}$ & $35(39)^{\dagger}$ \\
\hline Family history of CAD, $\mathrm{n}(\%)$ & $5(8)$ & $12(25)^{\dagger}$ & $8(9)$ \\
\hline Smoking, n (\%) & $4(6)$ & $4(8)$ & $39(44)^{*, \dagger}$ \\
\hline \multicolumn{4}{|l|}{ Medication } \\
\hline \multicolumn{4}{|l|}{ Pre-event medication } \\
\hline Without previous treatment, $\mathrm{n}(\%)$ & $46(73)$ & $5(10)^{\dagger}$ & $27(30)^{\star,+}$ \\
\hline Aspirin, $n(\%)$ & $4(6)$ & $32(67)^{\dagger}$ & $41(46)^{*, \dagger}$ \\
\hline ACE inhibitors, $n(\%)$ & $9(14)$ & $24(50)^{\dagger}$ & $30(34)^{\dagger}$ \\
\hline$\beta$ blockers, $\mathrm{n}(\%)$ & $3(5)$ & $22(46)^{\dagger}$ & $26(24)^{*, \dagger}$ \\
\hline Statins, $\mathrm{n}(\%)$ & $10(16)$ & $37(77)^{\dagger}$ & $35(39)^{\star,+}$ \\
\hline \multicolumn{4}{|l|}{ Postevent medication } \\
\hline Aspirin, n (\%) & - & $28(58)$ & 77 (87) \\
\hline ACE inhibitors n (\%) & - & $12(25)$ & $64(72)^{*}$ \\
\hline GP IIb/Illa inhibitors, n (\%) & - & $24(50)$ & $78(88)^{\star}$ \\
\hline ADP-receptor inhibitors, n (\%) & - & $4(8)$ & $44(49)^{\star}$ \\
\hline$\beta$ blockers, $\mathrm{n}(\%)$ & - & $8(17)$ & $60(67)^{*}$ \\
\hline Statins, n (\%) & - & $26(54)$ & $72(81)$ \\
\hline
\end{tabular}

Abbreviations: $A C E$, angiotensin-converting enzyme; ADP, adenosine diphosphate; AMl, acute myocardial infarction; BMl, body mass index; CAD, coronary artery disease; GP Illb/lla, glycoprotein Ilb/llla; SA, stable angina.

Data expressed as median and interquartiles (Q25-Q75), except when otherwise indicated

${ }^{*} P<0.05$ vs $S A$ group.

${ }^{\dagger} P<0.05$ vs CTR group.

\section{MATERIALS AND METHODS}

Study groups. A total of 200 subjects were recruited from cardiology service and from the outpatient clinic of cardiovascular risk in Santa Marta Hospital (Lisbon, Portugal; Table I): (1) 89 AMI patients (with documented ST-elevation changes, creatine kinase $[\mathrm{CK}]>3$ times normal and troponin positive) undergoing percutaneous coronary intervention (PCI) as reperfusion therapy were enrolled during the first 6 hours of the onset of chest pain (hospital admission); (2) 48 age- and sex-matched patients with SA pectoris, presenting typical chest discomfort and/or positive stress tests, which were submitted to coronary angiography; and (3) 63 healthy volunteers (CTR), with negative stress test, absence of any history of coronary disease, life-threatening diseases, or any other disease or condition that would impair compliance. These volunteers were not submitted to coronary angiography.

Patients' exclusion criteria included age $>85$ years; significant comorbidities as peripheral artery disease or carotid artery disease; known antecedents of malig- nant, infectious, and concurrent inflammatory diseases; chronic renal insufficiency; and previous myocardial infarction during the previous 5 years.

Core laboratory blood analysis for conventional tests and clinical chemistry, including $\mathrm{N}$-terminal pro-brain natriuretic peptide, $\mathrm{C}$-reactive protein, $\mathrm{CK}$, and cardiac troponin were measured in all patients and controls (Table II).

Study protocol and blood sampling. The AMI and SA patients were monitored at 2 time points: day 0 before PCI intervention and the administration of therapy, such as antithrombotic agents and IIb/IIIa inhibitors; 1 month after PCI. This protocol was designed to evaluate AMI patients at the acute phase of AMI (rupture and coronary occlusion) and at the early recovery phase (left ventricular remodeling), 1 month after. Previous studies indicated that the influence of medication in the values of inflammatory markers lasted for several days after PCI. ${ }^{22,23}$ Therefore, patients' assessment at 1-month evaluation represents the period of time for medication and clinical stabilization. 
Table II. Biochemical data in the studied population groups

\begin{tabular}{|c|c|c|c|c|c|}
\hline & \multirow[b]{2}{*}{ CTR } & \multicolumn{2}{|c|}{ SA } & \multicolumn{2}{|c|}{ AMI } \\
\hline & & Admission & 1 month & Admission & 1 month \\
\hline \multicolumn{6}{|l|}{ Inflammatory markers } \\
\hline $\mathrm{CRP}(\mathrm{mg} / \mathrm{dL})$ & $3.1(1.2-3.5)$ & $3.2(1.7-5.9)$ & $1.4(1.1-4.1)$ & $6.3(3.2-12)$ & $3.2^{\star}(3.1-6.7)$ \\
\hline $\mathrm{sCD} 40 \mathrm{~L}(\mathrm{ng} / \mathrm{mL})$ & $4.1(2.2-6.5)$ & $1.1^{\dagger}(0.68-2.2)$ & $5.6^{+, \neq}(3.0-8.5)$ & $1.3^{\dagger}(0.73-3.4)$ & $2.0^{*, \dagger}(0.79-3.3)$ \\
\hline \multicolumn{6}{|l|}{ Vascular function marker } \\
\hline VEGF $(\mathrm{pg} / \mathrm{mL})$ & $419(212-758)$ & $18^{\dagger}(1.1-295)$ & $293^{\ddagger}(192-442)$ & $48^{\dagger}(0.27-266)$ & $275^{\star}(161-493)$ \\
\hline \multicolumn{6}{|l|}{ Cardiac function markers } \\
\hline CK (U/L) & $111(80-195)$ & $82(58-107)$ & $97(35-159)$ & $315^{\dagger, \S}(113-1062)$ & $79 *(65-118)$ \\
\hline $\mathrm{cTnT}(\mathrm{ng} / \mathrm{mL})$ & $<0.01^{\|}$ & $<0.01^{\|}$ & $<0.01^{\|}$ & $0.33^{+, \S}(0.07-2.4)$ & $<0.01^{\|}$ \\
\hline NT-proBNP (pg/mL) & $38(16-64)$ & $98(51-247)$ & $155(55-424)$ & $356^{\dagger, \S}(145-1577)$ & $637^{*}(618-1404)$ \\
\hline
\end{tabular}

Abbreviations: AST, aspartate transaminase; AMI, acute myocardial infarction; CTnT, cardiac troponin T; CRP, C-reactive protein; CK, creatine kinase; NT-proBNP, N-terminus pro-B-type natriuretic peptide; SA, stable angina; SCD40L, soluble CD40 ligand; VEGF, vascular endothelial growth factor.

Data are expressed as median and quartiles (lower $25 \%$ quartile-upper $75 \%$ quartile). N.D.

${ }^{\star} P<0.05 \mathrm{LME}$ for AMl group variations over time.

${ }^{\dagger} P<0.05$ vs Control group.

${ }^{\ddagger} P<0.05 \mathrm{LME}$ for SA group variations over time.

${ }^{\S} P<0.05$ vs $S A$ group at hospital admission.

"Values below detection limit.

The study was conducted according to the principles expressed in the Declaration of Helsinki. The Ethical Committee Board of Centro Hospitalar de Lisboa Central approved the investigation and the protocol. All patients and volunteers enrolled signed a written consent following a full explanation of the study.

Blood samples were drawn into blood collection tubes without additives. For AMI and SA patients at hospital admission, the blood was collected immediately before PCI. For the following time point and for healthy volunteers (CTR), fasting blood samples were collected in early morning to avoid possible circadian variations. ${ }^{24}$

The serum was collected after centrifugation $(500 \mathrm{~g}$ for 10 minutes) within 15 minutes after sampling. Aliquots were stored at $-80^{\circ} \mathrm{C}$ until further analysis (no longer than 6 months). Samples were thawed only once.

Soluble CD4OL determination. Concentrations of SCD40L were measured in serum by enzyme-linked immunosorbent assays (ELISA) commercial kit (R\&D Systems). Each sample was measured in duplicate. The intra-assay variation among the duplicates for all samples was $<10 \%$, and concentrations were expressed in $\mathrm{ng} / \mathrm{mL}$.

The choice of serum to determine sCD40L by ELISA in this study had into consideration published data, ${ }^{25,26}$ and exploratory analysis performed by us. Ahn et $\mathrm{al}^{25}$ demonstrated that as long as preanalytical conditions were kept $<4^{\circ} \mathrm{C}$ neither blood origin (venous and arterial) nor blood fraction could significantly modify analytical results. However, Varo et $\mathrm{al}^{26}$ reported higher SCD40L concentrations in serum than those in plasma. One of the sources contributing to higher serum levels is platelets, therefore, poor-platelet plasma is recommended. ${ }^{25}$
We have performed $\mathrm{SCD} 40 \mathrm{~L}$ determinations on random blood samples $(n=42)$ on both serum and poor-platelet plasma fractions. For almost $40 \%$ of the analyzed samples $(\mathrm{n}=15)$, sCD40L concentrations in poor-platelet plasma were below the detection limit of the ELISA assay, contrasting with none in serum. Therefore, in the present study, the blood fraction used was serum. To safeguard sCD40L, stability temperature was kept at $4^{\circ} \mathrm{C}$ in all steps after blood collection, that is, transport and processing.

Platelet activation. Platelet activation was assessed by flow cytometry in whole blood samples within 3 hours after sampling as described previously. ${ }^{27}$ Blood was drawn into sodium-citrated blood collection tubes under minimal tourniquet pressure to avoid artifact platelet activation. A fluorescein isothiocyanateconjugate anti-CD42a antibody (BD) was used as an activation-independent marker of platelets. The expression of P-selectin was assessed by allophycocyanin-conjugate anti-CD62P antibody (BD). Platelets were identified on the basis of size and of fluorescein isothiocyanate-CD42a binding.

Microparticles identification. Microparticles were identified using an adjustment to the method previously described by Bernal-Mizrachi et al. ${ }^{28}$ In brief, plateletpoor plasma, obtained by double whole blood centrifugation, was incubated with fluorochromelabeled antibodies, phosphate-buffered saline (PBS) was added, and the sample was set to flow cytometry. Microparticles were identified on the basis of size defined by scattered light properties using platelets as an internal individual size standard in each sample (Fig. 1). ${ }^{29}$ The flow cytometer calibration and the 
A

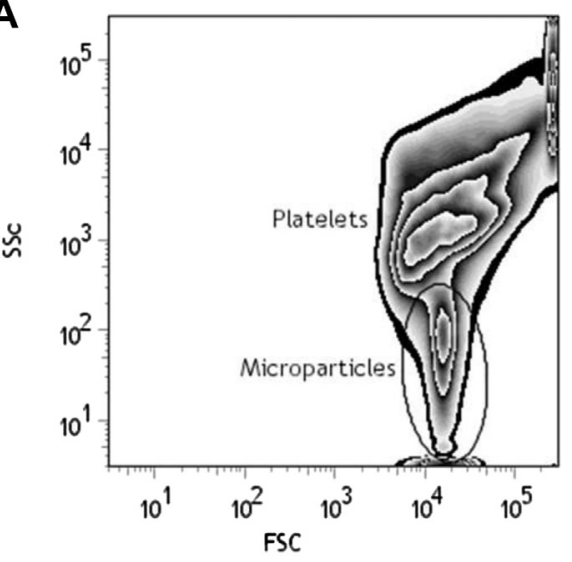

C

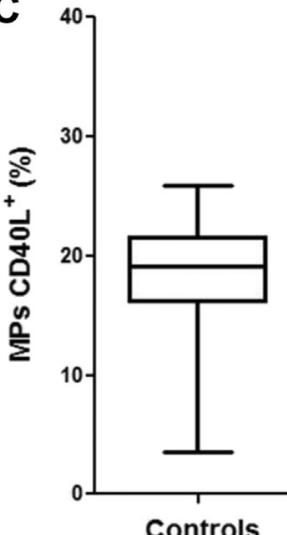

SA
B
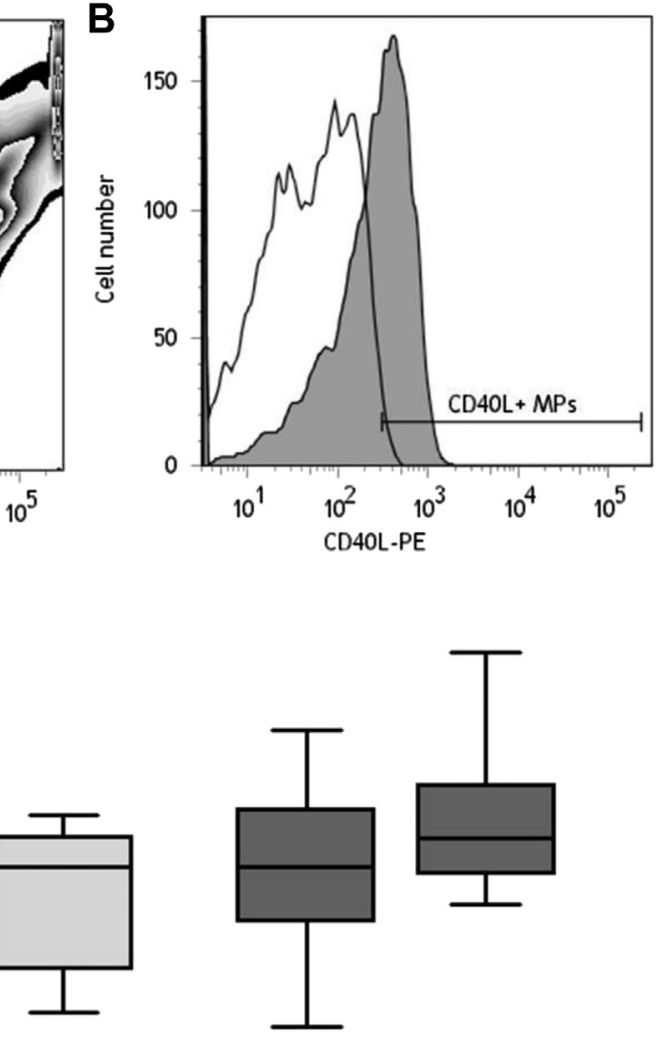

Admission

AMI

Fig 1. Identification of microparticles. (A) Representative flow cytometry zebra plot (contour and density) showing the region within elliptic area "Microparticles" localized below "Platelets," defined by scattered light properties (FSC vs SSC). (B) Representative flow cytometry fluorescence histogram showing the surface CD40L-positive microparticles gated on microparticles region. (C) Box-and-whisker plot of the percentage of CD40L-positive microparticles for controls, SA, and AMI patients. Plots depict the dispersion of the numeric values (box, 25\%-75\% interquartile; horizontal line, median; whiskers, minimum and maximum values). AMI, acute myocardial infarction; FSC, forward scattered light; MPs, microparticles; PE, phycoerythrin; SA, stable angina; SSC, side scattered light.

microparticles gate were set using polystyrene microspheres standards (Bangs Laboratories, Inc.) of $0.4 \mu \mathrm{m}$ and $0.69 \mu \mathrm{m}$ of nominal diameter. ${ }^{30,31}$ The microparticles gate was positioned below platelets, which were then used in each sample as a size internal control. Only the events falling in this gate were analyzed for fluorescence.

CD40L expression on platelets and microparticles. CD40L surface expression on platelets and microparticles was assessed by flow cytometry using PE-CD154 (BD).

Flow cytometry analysis. Flow cytometry was performed on a FACSCanto (BD), and data processed with FlowJo 7.6.5 (Tree Star Inc.). All samples were analyzed using the same voltage settings, and the instrument performance was daily monitored using BD CaliBRITE beads (BD). After correction for nonspecific binding, CD154 and CD62P expression were presented in fluorescence arbitrary units. Microparticles data were expressed in percentage of positive events.

Endothelial and vascular function markers. Polymorphisms G894T and T786C of eNOS protein were analyzed as markers of endothelial function. Genomic DNA was extracted from peripheral blood cells collected in ethylenediaminetetraacetic acid (EDTA) tubes using a PureLink Genomic DNA Mini Kit (Invitrogen). A region containing each polymorphism $^{32}$ was amplified by PCR using $1 \mathrm{mg}$ of DNA and $1 \mathrm{mM}$ of specific primers. Amplicons were then digested with specific restriction enzymes, and the digested fragments were visualized in a $2 \%$ ethidium bromide agarose gel.

The marker of vascular function chosen in this study was VEGF. Serum concentrations of this growth factor 
were measured by specific ELISA assays (designed to measure human VEGF-A) using the Quantikine Human VEGF kit (R\&D Systems) according to the manufacturer's protocol. Each sample was measured in duplicate; the intra-assay variation among the duplicates for all samples was $<10 \%$. The concentrations of VEGF were expressed in $\mathrm{pg} / \mathrm{mL}$.

Statistical analysis. Data were summarized as median and interquartiles 25\% and 75\% (Q25-Q75) for continuous variables and as proportions for categorical variables. Noncontinuous variables were analyzed using a $2 \times 2$ table and Fisher exact test. General linear model ANOVA with Bonferroni correction was used for continuous variables.

In AMI and SA groups, blood markers were measured in the same patient repeatedly in 2 different time points. Therefore, the set of observations are intercorrelated, and appropriate statistical methods were mandatory. A linear mixed effects (LMEs) model was applied. This statistical model describes the longitudinal variations of each patient allowing to estimate differences in average slopes between baseline (day 0 ) and the other time point, giving a measure of the variation of each blood marker over time. To apply LME, a logarithm transformation was applied to sCD40L, CD40L expression on platelets and microparticles, C-reactive protein, cardiac troponin, $\mathrm{N}$-terminal pro-brain natriuretic peptide, aspartate transaminase, and $\mathrm{CK}$, whereas a square root transformation was applied to VEGF.

The LME model was also used to assess the correlations between sCD40L concentrations and CD40L expression over time and other blood markers, demographic and clinical characteristics, and ongoing therapy.

Linear regression analysis was used to estimate the effect of confounders (risk factors, previous medication, comorbidities, etc) on sCD40L levels for each population and each evaluation point. Each model was crossvalidated with the baseline model.

Values of $P<0.05$ were considered statistical significant. The calculations were performed using SPSS (version 22.0) and R (version 2.11.1) software.

\section{RESULTS}

Soluble concentrations of CD40L. To investigate the sCD40L changes over time in AMI patients, the concentrations were also measured in SA and CTR groups. Soluble CD40L concentrations were reduced in AMI and SA patients at admission compared with CTR (Table II). In AMI and SA patients, sCD40L concentrations increased to 1 month.

The models of the average time changes of sCD40L were highly significant in both AMI $(F=5.3$; $P=0.01)$ and SA $(\mathrm{F}=10.1 ; P=0.003)$. Although the
sCD40L longitudinal increase was significant in both AMI and SA patients, the time-changes profiles were different $(F=13.5 ; P=0.001)$, being the rise greater in SA patients that reach values similar to CTR (Table II).

In the 3 groups studied (AMI, SA and CTR), the concentrations of sCD40L measured at admission were not significantly influenced by demographics, risk factors, and comorbidities. In addition, these factors did not modify the average changes of sCD40L over 1 month in AMI and SA groups.

The medication intake is summarized in Table I. The percentage of variability of SCD40L associated to medication at each evaluation point was relatively small in AMI patients and only significant at D30 $=34.8 \%$, $P=0.02$, being statins the major contributing factor exerting a positive effect on sCD40L levels. This weak influence of medication in SCD40L at discrete time points in AMI group did not reach significance when introduced in the regression model of sCD40L changes over time. Therefore, the average changes of sCD40L through time in AMI and SA patients were not altered by medication intake.

CD40L expression on platelets and microparticles. Platelets continue to be reported as the major source of $\mathrm{SCD} 40 \mathrm{~L}^{11,33}$ in spite previous studies had shown that platelets do not contain enough amount of $\mathrm{SCD} 40 \mathrm{~L}$ to be responsible for the circulating sCD40L. ${ }^{34}$ Recently studies considered SCD40L as a pool of free soluble and microparticle-bound forms. ${ }^{21}$ In this perspective, the CD40L expression on platelets and microparticles was assessed.

At hospital admission, the platelet CD40L expression in AMI patients was similar to that in SA and CTR groups (Fig 2). However, the values increased significantly to 1 month on AMI patients $(F=6.2 ; P=0.03$; Fig 2$)$.

The percentage of microparticles expressing CD40L was also determined. Although no major changes were verified in AMI comparing to SA and CTR groups, the $\mathrm{CD}_{40 \mathrm{~L}^{+}}$microparticles were also significantly increased to 1 month after PCI intervention $(F=6.2$; $P=0.03$; Fig 1).

Moreover, the expression of CD40L on platelets and on microparticles was not influenced by demographics, risk factors, and comorbidities or medication intake previous to admission.

The association of $\mathrm{sCD} 40 \mathrm{~L}$ with $\mathrm{CD} 40 \mathrm{~L}$ expression on platelets and microparticles were also evaluated in the studied groups. Soluble CD40L levels did not correlate with the expression of CD40L neither on platelets $(F<3.2 ; P>0.32$ and $r=0.27 ; P=0.90)$ nor on microparticles $(F<1.1 ; P>0.76$ and $r=0.39 ; P=0.10)$.

Associations of SCD4OL to VEGF and eNOS polymorphisms. To clarify the relationship of $\mathrm{SCD} 40 \mathrm{~L}$ with endothelial dysfunction and angiogenesis in AMI 

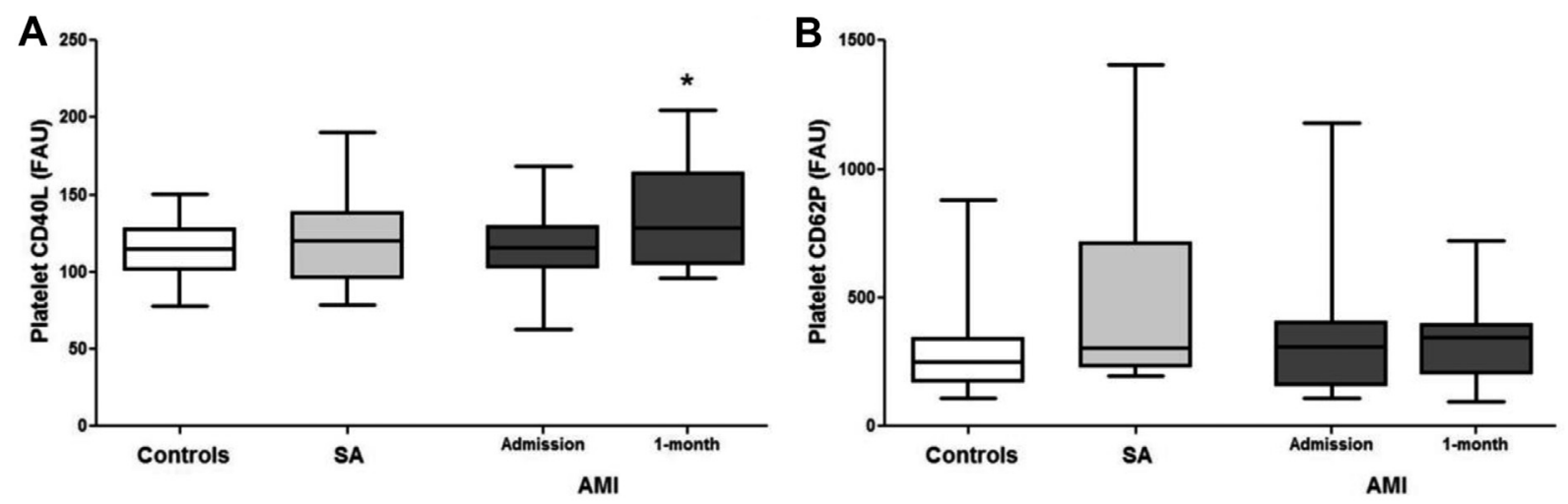

Fig 2. Expression of CD40L (A) and CD62P (B) on platelets for controls, SA, and AMI patients. $* P<0.05$ vs AMI group at hospital admission. Plots depicted the dispersion of the numeric values (box, $25 \%-75 \%$ interquartile; horizontal line, median; whiskers, minimum and maximum values). AMI, acute myocardial infarction; SA, stable angina; FAU, fluorescence arbitrary units.

patients, the eNOS polymorphisms and VEGF concentrations were assessed. As AMI and SA patients were monitored twice in 1 month, the LME regression model was used to assess correlations between soluble and bound CD40L, VEGF, and eNOS polymorphisms, whereas a Spearman correlation method was used in CTR group.

Noteworthy associations between $\mathrm{SCD} 40 \mathrm{~L}$ and endothelial and vascular function markers were obtained for AMI patients.

The G894T polymorphism of eNOS corresponds to a modification of the coding sequence $\left(\mathrm{Glu}^{298} \rightarrow\right.$ Asp) which results in the incorporation of aspartate in place of glutamate. ${ }^{35}$ The T786C polymorphism results in the replacement of thymine by cytosine. ${ }^{35}$

In our study, the eNOS G894T polymorphism was associated with the longitudinal variations of SCD40L in AMI $(F=6.9 ; P=0.01)$ but not in SA patients $(F=0.001$; $P=0.97)$ and in CTR subjects $(r=-0.35 ; P=0.79)$. No significant associations were observed between sCD40L and the eNOS T786C polymorphism in AMI $(F=0.21 ; P=0.65)$, SA $(F=0.14 ; P=0.72)$, or CTR groups $(r=-0.11 ; P=0.96)$.

Furthermore, sCD40L levels were correlated with the time-changes VEGF concentrations in AMI ( $F=9.9$; $P=0.02)$ and SA groups $(F=9.0 ; P=0.01)$. This statistical dependence was not verified in the CTR group, as far as monotonic relationship between paired data is concerned $(r=0.19 ; P=0.21)$.

The CD40L expression on platelets and on microparticles was not associated with either of the eNOS polymorphisms or of the VEGF concentrations in AMI, SA, or CTR groups.

Association of SCD40L to platelet CD62P expression. This study also intended to investigate the relation between SCD40L levels and markers of platelet activation, such as P-selectin (CD62P) expression.
No significant correlations were verified between sCD40L levels and the expression of CD62P on platelets in AMI, in SA patients $(F<1.3 ; P>0.42)$ and in CTR group $(r=-0.30 ; P=0.13)$.

\section{DISCUSSION}

In the present work, we studied whether SCD40L was related with markers of vascular function and of platelet activation along disease progression in AMI patients. Novel results were obtained highlighting the association between the time changes of SCD40L over 1 month after myocardial infarct onset and markers of endothelial and vascular function (G894T eNOS polymorphism and VEGF concentrations), but not to the platelet CD62P expression.

The time changes of sCD40L in AMI patients, along disease progression, correlate positively with the eNOS G894T polymorphisms, which is associated with endothelial dysfunction. The G894T polymorphism leads to a conservative replacement of glutamate with aspartate causing conformational alterations in the protein, thereby enhancing its susceptibility to proteolytic cleavage in endothelial cells and vascular tissues. ${ }^{36}$ Furthermore, this polymorphism has been associated with low plasma NO concentrations and with higher risk of CAD development. ${ }^{35,37}$ In our study, the longitudinal variations of sCD40L were correlated with this polymorphism, suggesting a relationship of G894T polymorphism with endothelial dysfunction along disease progression.

Moreover, the time changes of SCD40L also correlate with the variations of VEGF. VEGF is an endothelial cell-specific mitogen that has been reported to promote collateral vessels formation in ischemic cardiac muscle and tissue repair after injury. ${ }^{38}$ Circulating levels of 
VEGF could affect the outcome of AMI..$^{20,38,39}$ We have previously reported depressed serum VEGF concentrations immediately after AMI which increase over 1 year, ${ }^{20}$ with a similar trend to that observed in this study for sCD40L. The association between sCD40L and circulating VEGF was also described by Lim et $\mathrm{al}^{40}$ in CAD patients; however, in this case, the levels of both markers were increased in those patients. In addition, conflicting data were reported in studies using in vitro or animal models. Urbich et $\mathrm{al}^{10}$ observed a blockage of VEGF-induced angiogenesis by CD40L that could affect endothelial regeneration after plaque disruption. ${ }^{10}$ Other authors had shown that sCD40L transcriptionally regulates VEGF expression in endothelial cells, favoring growth and proliferation, and also promoting angiogenesis in mouse. ${ }^{41}$ Our results for AMI patients show a concomitant increase in sCD40L and VEGF levels along AMI progression. Therefore, the association of sCD40L with VEGF in AMI pathophysiology may suggest a role of sCD40L in angiogenesis.

The levels of sCD40L did not significantly correlate with the expression of platelet activation markers, such as CD62P. This may suggest that, along the disease progression in AMI patients, the SCD40L is not related to platelet activation or thrombosis.

Furthermore, no association of sCD40L with membrane-bound CD40L was found, which was not unexpected results. In fact, previous studies suggested that a variety of cells might be the source of SCD $40 \mathrm{~L},{ }^{42}$ what justifies the lack of correlation of SCD40L with a specific cell-type marker verified by us.

As far as cross-sectional testing is concerned, we observed that patients at hospital admission, regardless the acute or stable nature of coronary disease, showed remarkably low levels of sCD40L when compared with healthy subjects. After 1 month, the levels of sCD40L of SA patients rose to levels similar to those of CTR, whereas in AMI patients, the increase of sCD40L was also significant, but less marked. The differences between sCD40L concentrations in CAD (AMI and SA) patients at hospital admission and in controls could be related with the disparity in medication intake. Statins and combined antiplatelet therapy had been referred to lower sCD40L concentrations. ${ }^{43-47}$ However, in our study, there was no influence of preand postevent medication intake in the longitudinal variations of sCD40L levels, suggesting that the differences observed in the rate of SCD40L increase with time in AMI and SA patients were not related to medication intake. It can be hypothesized that low levels of sCD40L in CAD patients at hospital admission may reflect a persistent binding of CD40L. This may implicate a continuous activation of the signaling pathways in which CD40L is involved. The increase of sCD40L in AMI patients after 1 month proved to be slower than that in SA patients, probably reflecting the magnitude of injury and of involved territories.

In the last 2 decades intensive literature has been published reporting higher SCD40L in patients with both stable and unstable CAD (including AMI). 2,7-9,48-50 However, published results are difficult to compare. Time from disease onset to sample collection is often unspecified, which may cause strong deviations on absolute values of measured parameters. ${ }^{24-26}$ Nevertheless, we argue that in the present study, rigorous protocols were applied to mitigate sources of error originated from preanalytical and analytical methods.

Although the sCD40L had been implicated in the endothelial dysfunction and angiogenesis, ${ }^{3,10-14}$ there were no data available for AMI patients. Our results for AMI patients show a concomitant increase in sCD40L and VEGF levels along AMI progression and temporal variation of cardiac function markers toward stabilization. Therefore, the association of SCD40L with VEGF in AMI pathophysiology may suggest a role of $\mathrm{SCD} 40 \mathrm{~L}$ in endothelial dysfunction and angiogenesis. Our results show that, regarding AMI pathophysiology, sCD40L cannot be considered just as a marker of platelet activation. In fact, our results corroborate previous in vitro studies that implicate sCD40L in endothelial dysfunction and vascular function, demonstrating for the first time this association in a clinical observational study. The pathophysiologic implications of these findings are very important. A prognostic value for the sCD40L could be hypothesized along the progression of the disease in AMI patients. However, further studies are mandatory to clarify the time evolution of the link between SCD40L and VEGF in patients after AMI. Exploring this connection may support endothelial dysfunction assessment in AMI and eventually contribute to the establishment of a prognostic value to that link.

\section{ACKNOWLEDGMENTS}

Conflicts of Interest: The authors declare no conflicts of interest. All authors signing this article had read the journal's policy on conflicts of interest and the authorship agreement.

The work was financially supported by Fundação para a Ciência e Tencnologia (SFRM/BPD/6308/2009) and by Liga dos Amigos do Hospital de Santa Marta.

The article has been reviewed and approved by all the authors. 


\section{REFERENCES}

1. Mach F, Schönbeck U, Sukhova GK, et al. Functional CD40 ligand is expressed on human vascular endothelial cells, smooth muscle cells, and macrophages: implications for CD40-CD40 ligand signaling in atherosclerosis. Proc Natl Acad Sci U S A 1997;94:1931-6.

2. Aukrust P, Müller F, Ueland T, et al. Enhanced levels of soluble and membrane-bound CD40 ligand in patients with unstable angina: possible reflection of T lymphocyte and platelet involvement in the pathogenesis of acute coronary syndromes. Circulation 1999;100:614-20.

3. Muhammad R, Pathak D, Freedman JE, Chakrabarti S. CD40CD40 ligand interactions in oxidative stress, inflammation and vascular disease. Trends Mol Med 2008;14:530-8.

4. Santilli F, Basili S, Ferroni P, Davì G. CD40/CD40L system and vascular disease. Intern Emerg Med 2007:2:256-68.

5. Lutgens E, Lievens D, Beckers L, Donners M, Daemen M. CD40 and its ligand in atherosclerosis. Trends Cardiovasc Med 2007;17: $118-23$.

6. Zhang B, Wu T, Chen M, Zhou Y, Yi D, Guo R. The CD40/CD40L system: a new therapeutic target for disease. Immunol Lett 2013; 153:58-61.

7. Varo N, de Lemos JA, Libby P, et al. Soluble CD40L: risk prediction after acute coronary syndromes. Circulation 2003;108:1049-52.

8. Brueckmann M, Bertsch T, Lang S, et al. Time course of systemic markers of inflammation in patients presenting with acute coronary syndromes. Clin Chem Lab Med 2004;42:1132-9.

9. Kinlay S, Schwartz G, Olsson A, et al. Effect of atorvastatin on risk of recurrent cardiovascular events after an acute coronary syndrome associated with high soluble CD40 ligand in the myocardial ischemia reduction with aggressive cholesterol lowering (MIRACL) study. Circulation 2004;110:386-91.

10. Urbich C, Dernbach E, Aicher A, Zeiher AM, Dimmeler S. CD40 ligand inhibits endothelial cell migration by increasing production of endothelial reactive oxygen species. Circulation 2002;106: 981-6.

11. Furman MI, Krueger LA, Linden MD, Barnard MR, Frelinger AL III, Michelson AD. Release of soluble CD40L from platelets is regulated by glycoprotein IIb/IIIa and actin polymerization. J Am Coll Cardiol 2004;43:2319-25.

12. Chen C, Chai H, Wang X, et al. Soluble CD40 ligand induces endothelial dysfunction in human and porcine coronary artery endothelial cells. Blood 2008;112:3205-16.

13. Hristov M, Gümbel D, Lutgens E, Zernecke A, Weber C. Soluble CD40 ligand impairs the function of peripheral blood angiogenic outgrowth cells and increases neointimal formation after arterial injury. Circulation 2010;121:315-24.

14. Wolf D, Hohmann J-D, Wiedemann A, et al. Binding of CD40L to Mac-1's I-domain involves the EQLKKSKTL motif and mediates leukocyte recruitment and atherosclerosis-but does not affect immunity and thrombosis in mice. Circ Res 2011;109:1269-79.

15. Luo J-Q, Wen J-G, Zhou H-H, Chen X-P, Zhang W. Endothelial nitric oxide synthase gene G894T polymorphism and myocardial Infarction: a meta-analysis of 34 studies involving 21068 subjects. PLoS One 2014:9:e87196.

16. Çiftçi Ç, Melil S, Çebi Y, et al. Association of endothelial nitric oxide synthase promoter region (T-786C) gene polymorphism with acute coronary syndrome and coronary heart disease. Lipids Health Dis 2008:7:5.

17. Armstrong EJ, Morrow David A, Sabatine Marc S. Inflammatory biomarkers in acute coronary syndromes. Part III: biomarkers of oxidative stress and angiogenic growth factors. Circulation 2006;113:e289-92.
18. Di Marco GS, Reuter Stefan, Hillebrand Uta, et al. The soluble VEGF receptor sFlt1 contributes to endothelial dysfunction in CKD. J Am Soc Nephrol 2009;20:2235-45.

19. Petrovic D. The role of vascular endothelial growth factor gene as the genetic marker of atherothrombotic disorders and in the gene therapy of coronary artery disease. Cardiovasc Hematol Agents Med Chem 2010;8:47-54.

20. Ramos C, Napoleão P, Selas M, et al. Prognostic value of VEGF in patients submitted to percutaneous coronary intervention. Dis Markers 2014;201:135357.

21. Chung I, Choudhury A, Patel J, Lip GYH. Soluble CD40L, platelet surface CD40L and total platelet CD40L in congestive heart failure: relationship to platelet volume, mass and granularity. J Intern Med 2008;263:313-21.

22. Napoleão P, Santos MC, Selas M, Viegas-Crespo AM, Pinheiro T, Cruz Ferreira R. Variations in inflammatory markers in acute myocardial infarction: a longitudinal study. Rev Port Cardiol 2007;26:1357-63.

23. Napoleão P, Selas M, Freixo C, et al. The role of inflammatory biomarkers in the assessment of coronary artery disease. In: Baskot B, ed. Coronary angiography_advances in noninvasive imaging approach for evaluation of coronary artery disease. InTech, Rijeka, 2011.

24. Dominguez-Rodriguez A, Abreu-Gonzalez P, GarciaGonzalez MJ, Kaski JC. Diurnal variation of soluble CD40 ligand in patients with acute coronary syndrome. Soluble CD40 ligand and diurnal variation. Thromb Res 2009;123:617-21.

25. Ahn ER, Lander G, Jy W, et al. Differences of soluble CD40L in sera and plasma: implications on CD40L assay as a marker of thrombotic risk. Thromb Res 2004;114:143-8.

26. Varo N, Nuzzo R, Natal C, Libby P, Schönbeck U. Influence of pre-analytical and analytical factors on soluble CD40L measurements. Clin Sci 2006;111:341-7.

27. Monteiro MC, Sansonetty F, Gonçalces MJ, O'Connor JE. Flow cytometric kinetic assay of calcium mobilization in whole blood platelets using Fluo-3 and CD41. Cytometry 1999;35:302-10.

28. Bernal-Mizrachi L, Jy W, Fierro C, et al. Endothelial microparticles correlate with high-risk angiographic lesions in acute coronary syndromes. Int J Cardiol 2004;97:439-46.

29. Nieuwland R, Berckmans RJ, Rotteveel-Eijkman RC, et al. Cellderived microparticles generated in patients during cardiopulmonary bypass are highly procoagulant. Circulation 1997;96: 3534-41.

30. Dignat-George F, Sabatier F, Camoin-Jau L, Sampol J. Measuring circulating cell-derived microparticles. J Thromb Haemost 2004; 2:1844-5.

31. Shet AS, Key NS, Hebbel RP. Measuring circulating cell-derived microparticles. J Thromb Haemost 2004;2:1848-50.

32. Colombo MG, Paradossi U, Andreassi MG, et al. Endothelial nitric oxide synthase gene polymorphisms and risk of coronary artery disease. Clin Chem 2003;49:389-95.

33. Henn V, Steinbach S, Büchner K, Presek P, Kroczek RA. The inflammatory action of CD40 ligand (CD154) expressed on activated human platelets is temporally limited by coexpressed CD40. Blood 2001;98:1047-54.

34. Weber A-A, Hermann A, Rauch BH, Schrör K. Molecular identity of platelet CD40L ligand (CD40L). Thromb Haemost 2001;86: 718.

35. da Costa Escobar Piccoli J, Manfredini V, Hamester FI, et al. Interaction between endothelial nitric oxide synthase gene polymorphisms $(-786 \mathrm{~T}>\mathrm{C}, 894 \mathrm{G}>\mathrm{T}$ and intron $4 \mathrm{a} / \mathrm{b})$ and cardiovascular risk factors in acute coronary syndromes. Arch Med Res 2012;43:205-11. 
36. Tesauro M, Thompson WC, Rogliani P, Qi L, Chaudhary PP, Moss J. Intracellular processing of endothelial nitric oxide synthase isoforms associated with differences in severity of cardiopulmonary diseases: cleavage of proteins with aspartate vs. glutamate at position 298. Proc Natl Acad Sci U S A 2000;97:2832-5.

37. Hingorani AD, Liang CF, Fatibene J, et al. A common variant of the endothelial nitric oxide synthase (Glu298/Asp) is a major risk factor for coronary artery disease in the UK. Circulation 1999; 100:1515-20.

38. Hojo Y, Ikeda U, Zhu Y, et al. Expression of vascular endothelial growth factor in patients with acute myocardial infarction. J Am Coll Cardiol 2000;35:968-73.

39. Petrovic D, Verhovec R, Globocnik Petrovic M, Osredkar J, Peterlin B. Association of vascular endothelial growth factor gene polymorphism with myocardial infarction in patients with type 2 diabetes. Cardiology 2007;107:291-5.

40. Lim HS, Tayebjee MH, Tan KT, Patel JV, MacFadyen RJ, Lip GYH. Is soluble CD40 ligand a mediator of angiogenesis in patients with coronary artery disease? Thromb Res 2008;122: 307-13.

41. Melter M, Reinders MEJ, Sho M, et al. Ligation of CD40 induces the expression of vascular endothelial growth factor by endothelial cells and monocytes and promotes angiogenesis in vivo. Blood 2000;96:3801-8.

42. Blann AD, Choudhury A, Freestone J, Patel J, Lip GYH. Soluble CD40 ligand and atrial fibrillation: relationship to platelet activation, and endothelial damage/dysfunction. Int J Cardiol 2008;127: $135-7$.

43. Semb AG, van Wissen S, Ueland T, et al. Raised serum levels of soluble CD40 ligand in patients with familial hypercholesterole- mia: downregulatory effect of statin therapy. J Am Coll Cardiol 2003;41:275-9.

44. Alber HF, Frick M, Suessenbacher A, et al. Effect of atorvastatin on circulating proinflammatory T-lymphocyte subsets and soluble CD40 ligand in patients with stable coronary artery disease-a randomized, placebo-controlled study. Am Heart J 2006;151:139.

45. Santini E, Madec S, Corretti V, Ferrannini E, Solini A. Effect of statins on soluble CD40 ligand in hypercholesterolemic type 2 diabetic patients. J Endocrinol Invest 2008;31:660-5.

46. Park MW, Her SH, Cho JS, et al. Triple antiplatelet therapy (aspirin, clopidogrel, cilostazol) loading followed by maintenance reduces greater serum level of SCD40L and TNF-a than dual antiplatelet therapy in patients with acute myocardial infarction undergoing PCI: two-center, prospective, open label, randomized, pilot study. Circulation 2013;128:A16074.

47. Heeschen C, Dimmeler S, Hamm CW, et al. Soluble CD40 ligand in acute coronary syndromes. N Engl J Med 2003;348: 1104-11.

48. Yan JC, Zhu J, Gao L, et al. The effect of elevated serum soluble CD40 ligand on the prognostic value in patients with acute coronary syndromes. Clin Chim Acta 2004;343:155-9.

49. Schönbeck U, Varo N, Libby P, Buring J, Ridker PM. Soluble CD40L and cardiovascular risk in women. Circulation 2001; 104:2266-8.

50. Youssef AA, Chang LT, Sheu JJ, et al. Association between circulating level of CD40 ligand and angiographic morphologic features indicating high-burden thrombus formation in patients with acute myocardial infarction undergoing primary coronary intervention. Circ J 2007;71:1857-61. 